\title{
Apantallamiento gravitatorio y agujeros negros
}

\section{Gravitational screening and black holes}

\author{
Enrique Álvarez Vita
}

http://dx.doi.org/10.21503/CienciayDesarrollo.2015.v18i2.09

\section{RESUMEN}

En el presente trabajo proponemos un modelo físico en el que las partículas y antipartículas del vacío cuántico, que a su vez contendrían subniveles de vacío cuántico, polarizarían las cargas eléctricas y de color de leptones y quarks, en una secuencia infinita convergente, incluyendo la gravedad, estableciendo una nueva métrica espaciotemporal, lo que permitiría su incorporación a las demás fuerzas fundamentales de la naturaleza.

Palabras clave: Polarización cuántica, vacío cuántico fractal, antimateria virtual, métrica de Schwarzchild modificada.

\section{ABSTRACT}

Here we propose a physical model in which particles and anti particles of the quantum vacuum (which in turn contains sublevels) polarize the electric and color charges of quarks and leptons within an infinite convergent sequence inclusive of gravity, establishing a new spacetime metric and allowing to incorporate gravity to the other fundamental forces of nature.

Key words: Quantum polarization, fractal quantum vacuum, virtual anti matter, modified Schwarszchild metric.

\section{INTRODUCCIÓN}

El vacío cuántico contiene partículas virtuales de materia y antimateria. Proponemos que este vacío cuántico contiene a su vez subniveles fractales de partículas virtuales de materia y antimateria que interactúan con la partícula real en una secuencia infinita convergente, representada matemáticamente por una función analítica $\mathrm{f}(\mathrm{x})$.
Supongamos una partícula como el electrón. En el modelo estándar se considera al electrón como una partícula puntual sin dimensión.

Se ha demostrado experimentalmente que el electrón atrae positrones virtuales del vacío cuántico que atenúan su carga eléctrica.

Proponemos que estos positrones virtuales atraen a su vez electrones virtuales de un primer

${ }^{\star}$ Presidente del Centro de Estudios Aeroespaciales y Ciencias del Espacio. Vicepresidente de la Asociación Peruana de Filosofía y

Ciencia. Miembro de la Asociación Peruana de Historia de la Ciencia. Email: ejualvi@hotmail.com 
subnivel del vacío cuántico, incrementando la carga del electrón.

Estos electrones virtuales atraen positrones virtuales de un segundo nivel que atenúa la carga eléctrica, y así sucesivamente en una secuencia infinita.

La energía intrínseca del electrón está dada por $E=m_{e} c^{2}$, siendo $m_{e}$ la masa del electrón y c la velocidad de la luz en el vacío.

Si consideramos al electrón como una esfera imaginaria, la energía entre dos puntos a y b del centro de la partícula estará dada por:

$$
E_{a b}=A[f(b)-f(a)]
$$

Siendo A un coeficiente por determinar. Si a $\rightarrow 0$ y $b \rightarrow \infty$, tendremos:

$$
E=m_{e} c^{2}(1-0)
$$

Es decir, $A=m_{e} c^{2}, \mathrm{f}(\mathrm{a})=0$ y $\mathrm{f}(\mathrm{b})=1$.

Ahora bien, la única función que satisface la ecuación es la función exponencial $e^{-\lambda / r}$, cuando $r \rightarrow 0$ y $r \rightarrow \infty$, siendo $\lambda$ una constante de longitud por definir. La energía del cascarón imaginario a una distancia $r$ desde el infinito será:

$$
E=m_{e} c^{2}\left(1-e^{-\frac{\lambda}{r}}\right)
$$

Derivando la ecuación respecto a $\mathrm{r}$ obtendremos la fuerza del cascarón en $r$ :

$$
\boldsymbol{F}=\frac{m_{e} c^{2} \lambda}{r^{2}} e^{-\frac{\lambda}{r} \boldsymbol{r}}
$$

Siendo $\mathbf{r}$ un vector radial unitario dirigido desde el centro de la partícula.

Ahora bien, en la teoría clásica la fuerza electrogravitatoria autoinducida del electrón a una distancia $r$ está dada por la fuerza de repulsión eléctrica del electrón menos la fuerza de atracción gravitatoria, esto es:

$$
\boldsymbol{F}=\frac{k e^{-^{2}}}{r^{2}} \boldsymbol{r}-\frac{G m_{e}{ }^{2}}{r^{2}} \boldsymbol{r}
$$

Donde $\mathbf{k}$ es la constante electrostática coulombiana y e- la carga eléctrica del electrón.

A escalas de Planck, se puede utilizar el concepto de la fuerza gravitatoria newtoniana en lugar de la gravedad relativista.

Pero la derivada de la energía contiene el factor exponencial $e^{-\lambda / r}$ de manera que la ecuación de la fuerza sería:

$$
\boldsymbol{F}=\frac{k e^{-^{2}}-G m_{e}{ }^{2}}{r^{2}} e^{-\frac{\lambda}{r} \boldsymbol{r}}
$$

Igualando esta relación con la ecuación de la energía obtenemos:

$$
\lambda=\frac{k e^{-^{2}}-G m_{e}^{2}}{m_{e} c^{2}}
$$

Reemplazando este valor en las ecuaciones de la energía y fuerza electrogravitatoria autoinducida del electrón en $r$ tendremos:

$$
\begin{gathered}
E=m_{e} c^{2}\left(1-e^{\left.-\frac{k e^{-2}-G m_{e}^{2}}{m_{e} c^{2} r}\right)}\right. \\
F=\frac{k e^{-2}-G m_{e}^{2}}{r^{2}} e^{-\frac{k e^{-2}-G m_{e}^{2}}{m_{e} c^{2} r} r} r
\end{gathered}
$$

Cuyas gráficas sin escala comparadas con las del modelo clásico son respectivamente:

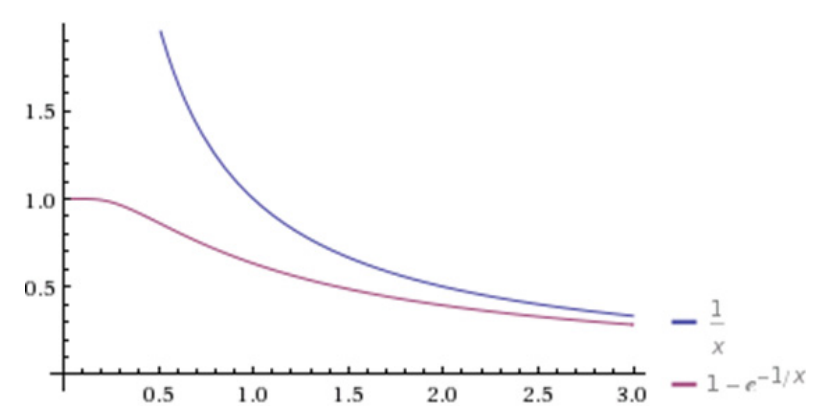




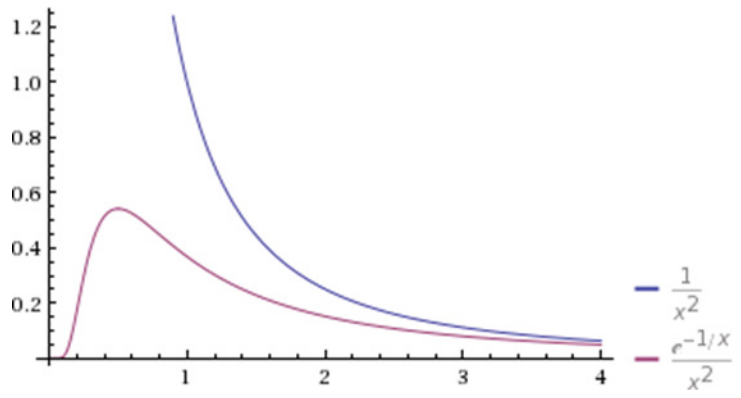

En el modelo clásico la energía tiende a infinito cuando $r \rightarrow 0$, mientras que en el modelo propuesto tiende a un valor finito $E=m_{e} c^{2}$ cuando $\mathrm{r} \rightarrow 0$, como muestra la gráfica, que es el valor observado experimentalmente.

Asimismo en el modelo clásico, la fuerza tiende a infinito cuando $r \rightarrow 0$, como muestra la gráfica. Este resultado infinito obligó a los físicos a recurrir al método de la renormalización.

Según nuestro modelo, la fuerza autoinducida del electrón se anula en el centro por efecto de las partículas virtuales de antimateria.

Obsérvese que para distancias muy cortas, la fuerza aumenta con la distancia, que es precisamente el comportamiento de las cargas de color.

En anteriores publicaciones sobre el modelo propuesto, partimos de la fuerza autoinducida del electrón en lugar de la energía, obteniendo el mismo resultado.

Si las ecuaciones de la energía y fuerza autoinducida del electrón las dividimos por su masa y carga eléctrica, es decir, $E / m_{\theta}$ y $\boldsymbol{F} / e^{-}$, obtendremos el potencial $\mathrm{V}$ y la intensidad $\mathrm{E}$ del campo eléctrico del electrón respectivamente.

\section{Laplaciano del potencial}

El laplaciano $\nabla^{2} \mathrm{~V}$ o divergencia del gradiente del potencial electrogravitatorio $\mathrm{V}$ el electrón a una distancia $r$ está dado por:

$$
\nabla^{2} V=\left(\frac{\partial^{2}}{\partial x^{2}}+\frac{\partial^{2}}{\partial y^{2}}+\frac{\partial^{2}}{\partial z^{2}}\right)\left[c^{2}\left(1-e^{-\frac{k e^{-2}-G m_{e}{ }^{2}}{m_{e} c^{2} r}}\right)\right]
$$

$$
=-\frac{\left(k e^{-^{2}}-G m_{e}^{2}\right)^{2}}{m_{e} c^{2} r^{4}} e^{-\frac{k e^{-^{2}}-G m_{e}^{2}}{m_{e} c^{2} r}}
$$

El significado físico de este resultado de signo negativo es que existe un sumidero en el fluido electrogravitatorio, más intenso en las proximidades del electrón, como consecuencia de la polarización cuántica, donde la energía negativa de las antipartículas virtuales apantallan la energía positiva electrogravitatoria.

En los modelos clásico y relativista, la divergencia es nula y en consecuencia no existe ni fuente ni sumidero, lo que conduce a valores infinitos en el modelo clásico y a una singularidad espaciotemporal en el modelo relativista, al no considerar los efectos de la polarización cuántica. Más adelante analizaremos el caso de la energía potencial gravitatoria de una masa.

Ahora bien, si consideramos que la constante de estructura fina del electrón a es $10^{43}$ mayor que la constante de acoplamiento gravitacional del electrón $\alpha_{g}$, podemos despreciar este término en las ecuaciones sin perder precisión, de manera que la intensidad del campo electrostático $\mathrm{E}$ del electrón en unidades gausianas, despreciando el campo gravitatorio extremadamente débil, estaría dada por:

$$
\boldsymbol{E}=\frac{e^{-}}{4 \pi \varepsilon_{0} r^{2}} e^{-\frac{\alpha \hbar}{m_{e} c r}} \boldsymbol{r}
$$

Que puede referirse también a una carga cualquiera $\mathrm{Q}$, siendo $\varepsilon_{0}$ la constante de permitividad en el vacío. La expresión $\alpha \hbar / m_{\theta} \mathrm{C}$ corresponde al radio clásico del electrón $r_{\theta}$.

A grandes distancias el exponencial tiende a la unidad y la ecuación se aproxima a la fórmula de la intensidad del campo electrostático de la física clásica. 


\section{Quarks}

Proponemos que el apantallamiento del electrón y demás leptones se aplica también a los quarks. Tratándose por ejemplo del quark up (u), con una carga eléctrica de $+2 \mathrm{e}^{-} / 3$, su energía será:

$$
E=m_{q_{u}} c^{2}\left(1-e^{-\frac{K \epsilon^{2}+\frac{4}{9} k e^{-2}-G m_{q_{u}}{ }^{2}}{m_{q_{u}} c^{2} r}}\right)
$$

En el caso del quark down (d), con carga eléctrica $-\mathrm{e}^{-} / 3$, su energía será:

$$
E=m_{q_{d}} c^{2}\left(1-e^{-\frac{K \epsilon^{2}+\frac{1}{9} k e^{-2}-G m_{q_{d}}{ }^{2}}{m_{q_{d}} c^{2} r}}\right)
$$

Siendo K una constante de proporcionalidad y $\epsilon$ la carga de color de los quarks.

Derivando respecto a $\mathrm{r}$ obtenemos una gráfica semejante a la fuerza electrogravitatoria autoinducida del electrón.

Para longitudes muy pequeñas, la fuerza aumenta con la distancia, que es precisamente el comportamiento de los quarks.

\section{Gravedad}

Para definir la gravedad según el modelo propuesto, lo aplicaremos primero por cuestiones metodológicas a la gravedad newtoniana, para luego aplicarlo a la relatividad general. Según las ecuaciones, la energía intrínseca de las partículas elementales se debe a su propia carga.

En el caso de los leptones a su carga electrostática y en el de los quarks a su carga de color.

No obstante, a distancias muy pequeñas, las cargas se anulan entre sí.

En el caso de los protones y neutrones, de acuerdo a la cromodinámica cuántica, las cargas de color de sus respectivos quarks se neutralizan dando como resultado el color blanco.
En el núcleo atómico conformado por varios protones y neutrones, la carga residual de la fuerza fuerte que interactúa entre los quarks también se neutraliza.

Asimismo, las cargas eléctricas de los quarks del neutrón se anulan, mientras que las del protón dan como resultado $+\mathrm{e}^{-}$, la cual se neutraliza con la carga $-\mathrm{e}^{-}$del electrón a nivel del átomo o a nivel molecular.

La carga gravitatoria es la única que no se anula, en consecuencia, siendo la más débil, su efecto acumulativo la convierte en la más poderosa a nivel del cosmos, no obstante se trata de una carga polarizada, lo que es fundamental para la comprensión de la gravedad a gran escala según nuestro modelo, como veremos a continuación.

\section{Potencial newtoniano modificado}

La sumatoria de la energía de las partículas elementales que constituyen la materia es la que confiere la energía a la masa.

Teniendo en cuenta que estas partículas polarizan la gravedad y considerando que podemos asumir una masa $\mathrm{M}$ como idealmente puntual para efectos de cálculo, de manera análoga al método empleado por Newton para calcular la gravedad de la Tierra y del sistema solar, podemos determinar, siguiendo el mismo procedimiento matemático que utilizamos con las partículas elementales, la energía gravitatoria autoinducida $\mathrm{E}$ de una masa cualquiera $M$ en un punto $r$, que está dada por:

$$
E=-M c^{2}\left(1-e^{-\frac{G M}{c^{2} r}}\right)
$$

El signo negativo de esta ecuación se debe a que la energía gravitatoria es siempre negativa, pero a diferencia de los modelos newtoniano y relativista, converge a una cantidad finita y no infinita cuando $r \rightarrow 0$, debido a la polarización cuántica de las partículas virtuales de antimateria, que es la energía intrínseca de la masa con signo negativo, 
lo que representa una posible solución al problema de la gravedad cuántica.

Dividiendo por $\mathrm{M}$ obtendremos el potencial newtoniano modificado $\mathrm{V}$ :

$$
V=-c^{2}\left(1-e^{-\frac{G M}{c^{2} r}}\right)
$$

Expandiendo el exponencial y despreciando la serie a partir del tercer término para grandes distancias, obtendremos el potencial newtoniano como caso particular.

Derivando con respecto a $r$ tendremos la aceleración gravitatoria $\mathbf{g}$ en $\mathrm{r}$ :

$$
\boldsymbol{g}=-\frac{G M}{r^{2}} e^{-\frac{G M}{c^{2} r} \boldsymbol{r}}
$$

Siendo $\mathbf{r}$ un vector unitario dirigido al centro de la masa.

Expandiendo el exponencial y despreciando la serie a partir del segundo término para distancias grandes, tendremos la aceleración gravitatoria newtoniana como caso particular.

Multiplicando ambas ecuaciones por una masa cualquiera $\mathrm{m}$ tendremos la energía y fuerza gravitatorias de $\mathrm{m}$ respecto a $M$ respectivamente, es decir, $E_{M_{m}}$ y $\boldsymbol{F}_{M_{m}}$

\section{Velocidad angular}

La velocidad angular $\omega$ estaría dada por:

$$
\omega=\frac{1}{r} \sqrt{\frac{G M}{r}} e^{-\frac{G M}{2 c^{2} r}}
$$

Este resultado significa que la velocidad angular es menor que la de la mecánica clásica. Cuando $r \rightarrow 0, \omega \rightarrow 0$.

Expandiendo el exponencial y despreciando términos obtendremos la velocidad angular de la mecánica clásica.

\section{Laplaciano del potencial}

El laplaciano $\nabla^{2} \mathrm{~V}$ del potencial newtoniano $\mathrm{V}$ al exterior de una masa $\mathrm{M}$ a una distancia $\mathrm{r}$ está dado por:

$$
\begin{gathered}
\nabla^{2} V=\left(\frac{\partial^{2}}{\partial x^{2}}\right. \\
\left.+\frac{\partial^{2}}{\partial y^{2}}+\frac{\partial^{2}}{\partial z^{2}}\right)\left[c^{2}\left(1-e^{-\frac{G M}{c^{2} r}}\right)\right] \\
=\frac{G^{2} M}{c^{2} r^{4}} e^{-\frac{G M}{c^{2} r}}
\end{gathered}
$$

Cuya gráfica sin escala es:

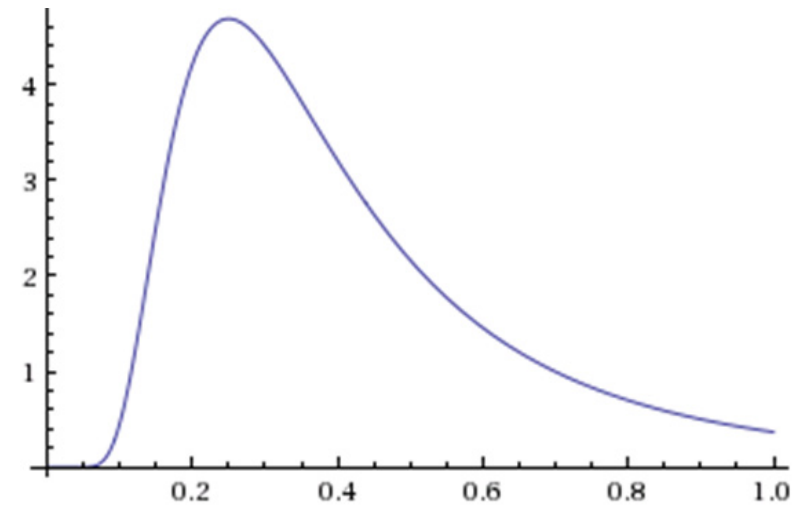

El significado físico de este resultado de signo positivo es que existe una fuente en el fluido gravitatorio, más intenso en las proximidades del centro de la masa puntual, como consecuencia de la polarización cuántica, donde la energía positiva gravitatoria de las partículas virtuales de antimateria apantallan la energía negativa gravitatoria.

En los modelos clásico y relativista, la divergencia es nula y en consecuencia no existe fuente, lo que conduce a valores infinitos en el modelo clásico y a una singularidad espaciotemporal infinita en el modelo relativista, al no considerar los efectos de la polarización cuántica. Más adelante analizaremos el caso relativista

\section{Al interior de una esfera sólida}

Podemos calcular la intensidad del campo gravitatorio $\mathbf{g}$ en el interior de una masa esférica sólida y homogénea $\mathrm{M}$ a una distancia $\mathrm{r}$. Teniendo en cuenta que:

$$
\frac{M}{m}=\frac{r_{0}{ }^{3}}{r^{3}}
$$


Siendo $\mathrm{r}_{0}$ el radio de $\mathrm{M}$ y $\mathrm{m}$ la masa concéntrica al interior de $\mathrm{M}$ de radio $\mathrm{r}$. De lo cual se deduce que:

$$
\boldsymbol{g}=-\frac{G M r}{r_{0}^{3}} e^{-\frac{G M r^{2}}{c^{2} r_{0}{ }^{3}}} \boldsymbol{r}
$$

Cuya gráfica sin escalas es:

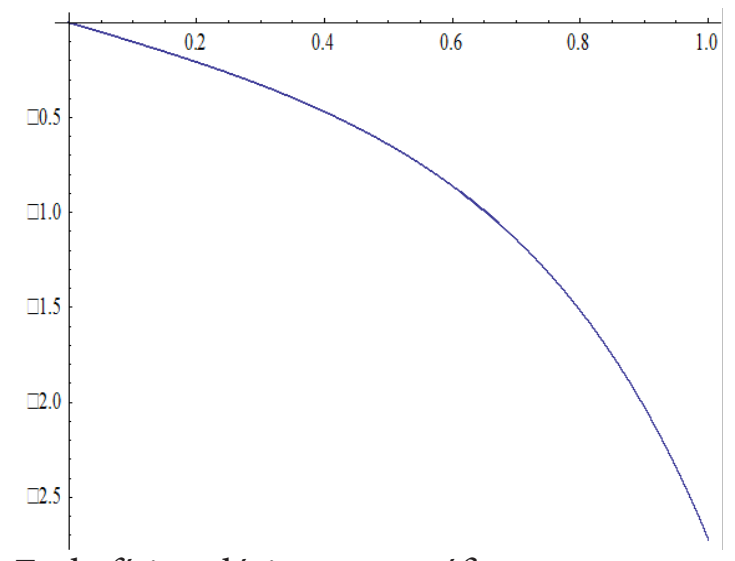

En la física clásica, esta gráfica es una recta.

El potencial gravitatorio de $\mathrm{V}$ es:

$$
V=\frac{c^{2}}{2}\left(1-e^{-\frac{G M r^{2}}{c^{2} r_{0}{ }^{2}}}\right)
$$

El laplaciano $\nabla^{2}$ de $\mathrm{V}$ está dado por:

$$
\begin{gathered}
\nabla^{2} V=\left(\frac{\partial^{2}}{\partial x^{2}}+\frac{\partial^{2}}{\partial y^{2}}+\frac{\partial^{2}}{\partial z^{2}}\right)\left[\frac{c^{2}}{2}\left(1-e^{-\frac{G M r^{2}}{c^{2} r_{0}{ }^{3}}}\right)\right] \\
=\frac{G M}{r_{0}{ }^{3}}\left(3-\frac{2 G M r^{2}}{c^{2} r_{0}{ }^{3}}\right) e^{-\frac{G M r^{2}}{c^{2} r_{0}{ }^{3}}}
\end{gathered}
$$

o bien:

$$
\nabla^{2} V=4 \pi G \rho\left(1-\frac{8 \pi G \rho r^{2}}{9 c^{2}}\right) e^{-\frac{4 \pi G \rho r^{2}}{3 c^{2}}}
$$

Siendo $\rho$ la densidad de $\mathrm{M}$.

Supongamos una esfera sólida homogénea de radio $r_{0}=1 \mathrm{~m}$. Las gráficas a escala de las intensidades de $\nabla^{2} \mathrm{~V}$ al interior de la esfera en los puntos $r_{1}=0,5 \mathrm{~m} . \mathrm{y} r_{2}=0,6 \mathrm{~m}$., por ejemplo, en función de la densidad $\rho$, son las siguientes:

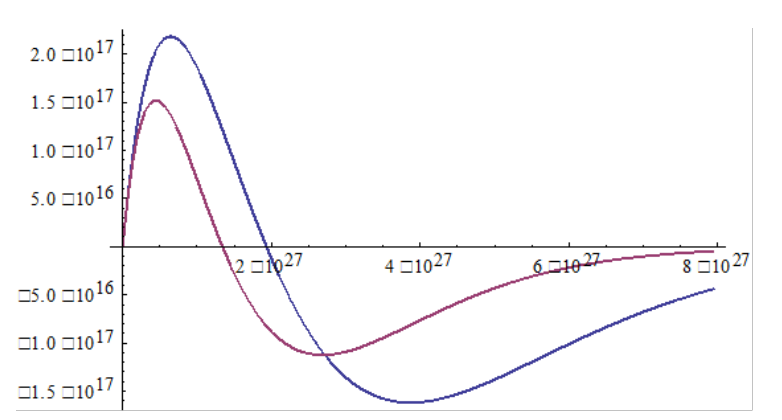

La gráfica de mayor intensidad de $\nabla^{2} \mathrm{~V}$ corresponde a $r_{1}$ y la de menor intensidad a $r_{2}$, es decir, a medida que el radio interior disminuye, el valor del laplaciano aumenta.

Se observa en las gráficas que para valores pequeños de densidad, el laplaciano es positivo y se incrementa a medida que la densidad aumenta hasta alcanzar un valor máximo, para luego disminuir hasta alcanzar un valor negativo mínimo, a partir del cual comienza a decrecer la intensidad hasta anularse, es decir, cuando $\rho \rightarrow \infty$, $\nabla^{2} \mathrm{~V} \rightarrow 0$.

Este resultado nos muestra lo que ocurriría al interior de una masa de gran densidad, como las estrellas de neutrones, y una mayor información sobre la naturaleza de los agujeros negros.

Cuando la densidad es relativamente baja, la ecuación se aproxima a:

$$
\nabla^{2} V=4 \pi G \rho
$$

Que es la ecuación de Poisson para el potencial gravitatorio newtoniano al interior de una esfera, como caso particular.

De modo análogo se calcula el laplaciano al interior de una esfera con carga eléctrica.

\section{Radio de escape del fotón}

El radio de escape de un fotón de masa relativista $\mathrm{m}$ de la gravedad de una masa $\mathrm{M}$, estaría dado, de modo análogo a la gravedad newtoniana, por la equivalencia entre sus energías cinética y gravitatoria, es decir: 


$$
\frac{m c^{2}}{2}=m c^{2}\left(1-e^{-\frac{G M}{c^{2} r}}\right)
$$

De donde el radio de escape ${ }^{\star}$ está dado por:

$$
r^{*}=-\frac{G M}{c^{2} \ln [1 / 2]}
$$

Siendo ln el logaritmo neperiano.

Si bien en la teoría de la relatividad la velocidad de la luz es constante y los fotones no se frenan por efecto de la gravedad, las ondas de luz sufren un corrimiento hacia el rojo por efecto de la curvatura espaciotemporal y se mantienen dentro de un horizonte determinado por del radio de Schwarzschild, como veremos más adelante.

\section{Métrica de Schwarzschild modificada}

Según la relatividad general, la gravedad es geometría y no una fuerza, definida por las ecuaciones tensoriales de Einstein.

Estas ecuaciones no tienen soluciones exactas sino aproximaciones.

La primera solución exacta la encontró Schwarzschild en el exterior de una distribución de materia con simetría esférica, asumiendo que toda la masa $\mathrm{M}$ está concentrada en $\mathrm{r}=0$.

En este caso, el elemento de línea en la métrica de Schwarzschild en coordenadas esféricas $(c t, r, \theta, \emptyset)$ donde $t$ es el tiempo medido por el observador, está dado por:

$$
\begin{gathered}
d s^{2}=-\left(1-\frac{2 G M}{c^{2} r}\right) c^{2} d t^{2}+\left(1-\frac{2 G M}{c^{2} r}\right)^{-1} d r^{2}+r^{2} d \theta^{2}+ \\
+r^{2} \sin ^{2} \theta d \emptyset^{2}
\end{gathered}
$$

Esta métrica se deduce a partir de la métrica de Minkowski con simetría esférica en ausencia de campo, es decir, sin presencia de masa:

$$
d s^{2}=-c^{2} d t^{2}+d r^{2}+r^{2} d \Omega^{2}
$$

Donde $\mathrm{d} \Omega^{2}=\mathrm{d} \theta^{2}+\sin \theta^{2} \mathrm{~d} \emptyset^{2}$, asumiendo el potencial newtoniano $-\mathrm{GM} / \mathrm{r}$.

Esta métrica determina la trayectoria relativista de un cuerpo de masa $\mathrm{m}$ que gira alrededor de otro cuerpo de masa $\mathrm{M}$.

Pero a nosotros nos interesa la aceleración radial aparente de una masa m cuya trayectoria se dirige directamente al centro de $\mathrm{M}$, que es precisamente la aceleración que experimenta una masa sobre sí misma y da origen a un agujero negro.

En consecuencia, si consideramos un biespacio $(t, r)$, tendremos $d \theta=d \emptyset=0$, por consiguiente el momento angular relativista es nulo y la métrica de Schwarzschild se reduce a:

$$
d s^{2}=-\left(1-\frac{2 G M}{c^{2} r}\right) c^{2} d t^{2}+\left(1-\frac{2 G M}{c^{2} r}\right)^{-1} d r^{2}
$$

Pero según el modelo propuesto, el potencial newtoniano está dado por $-c^{2}\left(1-e^{-G M / c^{2} r}\right)$, como señalamos anteriormente, de modo que, reemplazando este valor en la métrica de Schwarzschild en lugar del potencial newtoniano obtenemos una nueva métrica:

$$
d s^{2}=-\left(2 e^{-\frac{G M}{c^{2} r}}-1\right) c^{2} d t^{2}+\left(2 e^{-\frac{G M}{c^{2} r}}-1\right)^{-1} d r^{2}
$$

Expandiendo el exponencial y despreciando la serie a partir del tercer término para distancias relativamente grandes, obtendremos la métrica de Schwarzschild, como caso particular.

Cuando $r \rightarrow \infty$ obtenemos la métrica de Minkowski, coincidiendo con la métrica de Schwarzschild a una distancia situada en el infinito libre de campos gravitatorios.

Cuando $r \rightarrow 0$, obtenemos nuevamente una métrica de Minkowski pero con los signos cambiados, que puede representar una transformación recíproca entre las coordenadas espaciotemporales o el posible origen de un agujero blanco. 
Cabe señalar que como consecuencia del principio de simetría CPT (carga, imagen especular y tiempo), analizado con mayor detalle en anteriores publicaciones, el tiempo de las partículas virtuales de antimateria transcurre del pasado hacia el futuro, pero en sentido opuesto al de la materia, de manera que estas partículas virtuales neutralizan el retardo temporal relativista en la métrica de Schwarzschild.

Este nuevo resultado difiere sustancialmente de la relatividad general que se basa en el potencial newtoniano clásico y no en el potencial newtoniano modificado por las partículas virtuales de antimateria.

Con la nueva métrica eliminamos los infinitos de la singularidad espaciotemporal basada en la relatividad general cuando $r \rightarrow 0$, como veremos más adelante.

El nuevo tensor métrico sería entonces el siguiente:

$$
g_{u v}=\left[\begin{array}{cc}
-\left(2 e^{-\frac{G M}{c^{2} r}}-1\right) & 0 \\
0 & \left(2 e^{-\frac{G M}{c^{2} r}}-1\right)^{-1}
\end{array}\right]
$$

\section{Radio del horizonte de eventos}

De la expresión entre paréntesis de la métrica de Schwarzschild se deduce que:

$$
r=\frac{2 G M}{c^{2}}
$$

Conocido como radio de Shwarzschild, que determina el horizonte de eventos de un agujero negro, donde la velocidad de la luz o cualquier onda electromagnética queda atrapada dentro de ese horizonte, el cual es detectado por sus efectos gravitacionales, radio que coincide con el radio de escape del fotón en el modelo newtoniano.
De la expresión entre paréntesis de la métrica propuesta por nosotros se deduce que el radio del horizonte $\mathrm{r}^{\star}$ está dado por:

$$
r^{*}=-\frac{G M}{c^{2} \ln [1 / 2]}
$$

El cual coincide también con el radio de escape del fotón propuesto por nosotros en el modelo newtoniano.

El horizonte de eventos sería en consecuencia menor que el radio de Schwarzschild.

\section{Atraso temporal}

En la métrica de Schwarzschild el atraso temporal entre dos relojes $\mathrm{T}_{1} \mathrm{y} \mathrm{T}_{2}$ a distancias $r_{1}$ y $r_{2}$ respectivamente, está dado por:

$$
\Delta T_{2}=\Delta T_{1} \sqrt{\frac{1+2 \phi_{2} / c^{2}}{1+2 \phi_{1} / c^{2}}}
$$

Donde $\phi_{1}$ y $\phi_{2}$ son los potenciales newtonianos en $r_{1} \mathrm{y}_{2}$. Sustituyendo estos potenciales por los potenciales modificados tendremos:

$$
\Delta T_{2}=\Delta T_{1} \sqrt{\frac{e^{-G M / c^{2} r_{2}}-1}{e^{-G M / c^{2} r_{1}}-1}}
$$

\section{Corrimiento hacia el rojo}

Del mismo modo obtenemos el desplazamiento de las frecuencias $\mathrm{V}_{1}=1 / \Delta \mathrm{T} 1$ y $\mathrm{V}_{2}=1 / \Delta \mathrm{T} 2$ :

$$
v_{2}=v_{1} \sqrt{\frac{e^{-G M / c^{2} r_{1}}-1}{e^{-G M / c^{2} r_{2}}-1}}
$$

Si $r_{2}<r_{1}$, se tiene $v_{2}>v_{1}$, es decir, un corrimiento hacia el azul. Si $r_{2}>r_{2}$, entonces $v_{2}<v_{1} y$ tendremos un corrimiento hacia el rojo. 


\section{Aceleración radial aparente}

Para el caso de la nueva métrica el lagrangiano L está dado por:

$$
\mathcal{L}=c=\left[-\left(2 e^{-\frac{G M}{c^{2} r}}-1\right) c^{2} \dot{t}^{2}+\left(2 e^{-\frac{G M}{c^{2} r}}-1\right)^{-1} \dot{r}^{2}\right]^{\frac{1}{2}}
$$

De donde:

$$
c^{2}=-\left(2 e^{-\frac{G M}{c^{2} r}}-1\right) c^{2} \dot{t}^{2}+\left(2 e^{-\frac{G M}{c^{2} r}}-1\right)^{-1} \dot{r}^{2}
$$

Como $\partial \mathrm{L} / \partial \mathrm{t}=0$, entonces se tiene $\mathrm{d} / \mathrm{d} \tau(\partial \mathrm{L} / \partial \mathrm{t})=0$, siendo $\tau$ el tiempo propio. Es decir $\partial \mathrm{L} / \partial \dot{\mathrm{t}}=$ cte.

Dicha constante es la energía relativista por unidad de masa:

$$
\left(2 e^{-\frac{G M}{c^{2} r}}-1\right) \dot{t}=\frac{E}{m c^{2}}
$$

De donde:

$$
\dot{t}^{2}=\frac{E^{2}}{m^{2} c^{4}}\left(2 e^{-\frac{G M}{c^{2} r}}-1\right)^{-2}
$$

Cuando $r \rightarrow \infty$ entonces $\left(2 e^{-\frac{G M}{c^{2} r}}-1\right) \rightarrow 1$, de manera que nos encontramos en la métrica de Minkowski, donde $E=m c^{2} \gamma$, siendo $t=\gamma=1 / \sqrt{\left(1-v^{2} / c^{2}\right)}$.

Reemplazando $\dot{t}^{2}$ tendremos:

$c^{2}=-\left(2 e^{-\frac{G M}{c^{2} r}}-1\right)^{-1} \frac{E^{2}}{m^{2} c^{2}}+\left(2 e^{-\frac{G M}{c^{2} r}}-1\right)^{-1} \dot{r}^{2}$

Resolviendo la ecuación, restando $2 \mathrm{~m}^{2} \mathrm{c}^{4} \mathrm{y}$ dividiendo por $2 \mathrm{mc}^{2}$, obtendremos una nueva energía $\mathrm{E}^{*}$ :

$$
\begin{gathered}
\frac{E^{2}-m^{2} c^{4}}{2 m c^{2}}=E^{*}=m c^{2} e^{-\frac{G M}{c^{2} r}}+\frac{m \dot{r}^{2}}{2}-m c^{2} \\
\frac{m \dot{r}^{2}}{2}-m c^{2}\left(1-e^{-\frac{G M}{c^{2} r}}\right)
\end{gathered}
$$

Lo que nos da la energía potencial gravitatoria newtoniana modificada, despreciando $\mathrm{mr}^{2} / 2$, por tratarse del cuadrado de un diferencial.
Si consideramos, de modo análogo al caso de las partículas elementales, la energía gravitatoria autoinducida de $\mathrm{M}$, tendremos:

$$
E^{*}=-M c^{2}\left(1-e^{-\frac{G M}{c^{2} r}}\right)
$$

Cuando $\mathrm{r} \rightarrow 0, \mathrm{E}^{\star}=-\mathrm{Mc}^{2}$, que es la energía gravitatoria de un agujero negro de masa $\mathrm{M}$ según la nueva métrica, una magnitud finita equivalente a la energía intrínseca de $\mathrm{M}$ con signo negativo, a diferencia del valor infinito de la métrica relativista, como consecuencia de los efectos cuánticos en la relatividad general.

Este resultado nos conduce a la posibilidad de establecer un origen común para las fuerzas fundamentales de la naturaleza, incluyendo la gravedad, como gradientes diferenciados de una misma fuente que es la energía intrínseca $\mathrm{E}=\mathrm{Mc}^{2}$.

Dividiendo la expresión por $\mathrm{M}$ obtenemos el potencial gravitatorio modificado.

La gráfica sin escala a continuación muestra los potenciales gravitatorios relativista y modificado en función de la distancia al centro del agujero negro:

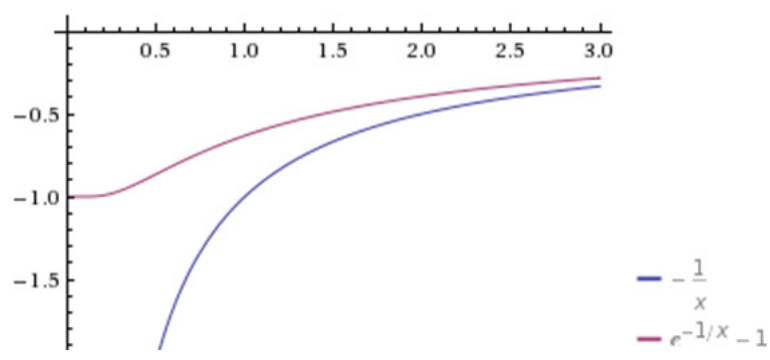

Cuando $r \rightarrow 0, V=-c^{2}$

Derivando $E^{\star}$ con respecto a $\tau$ tendremos:

$$
0=m \dot{r} \ddot{r}+\frac{G M m}{r^{2}} e^{-\frac{G M}{c^{2} r}} \dot{r}
$$

Es decir:

$$
\ddot{r}=-\frac{G M}{r^{2}} e^{-\frac{G M}{c^{2} r}}
$$

Que es precisamente la aceleración radial g:

$$
g=-\frac{G M}{r^{2}} e^{-\frac{G M}{c^{2} r} r}
$$


La gráfica sin escala a continuación muestra las aceleraciones aparentes relativista y modificada en función de la distancia al centro del agujero negro:

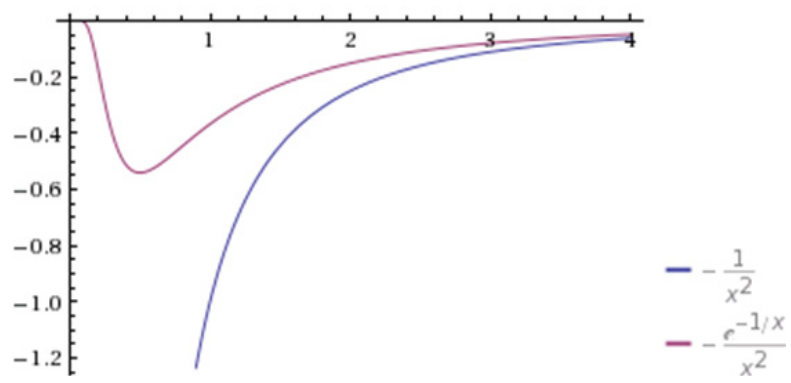

La métrica de Eddington - Finkelstein con un cambio de coordenadas elimina la singularidad cuando la distancia es igual al radio de Schwarzschild, lo que ocurre también con la nueva métrica.

La métrica de Kerr - Newuman para un agujero negro cargado en rotación y otras métricas las abordaremos en una próxima publicación.

\section{Expansión del universo}

Hemos visto que a una distancia nula obtenemos la métrica de Minkowski invertida, lo que podría representar un agujero blanco.

Si consideramos la totalidad del universo como isotrópico y homogéneo con una topología hiperesférica de cuatro dimensiones e invertimos el proceso, basado en la métrica de Minkowski invertida, tendremos un universo en expansión acelerada, como se ilustra en la figura, lo que podría explicar la energía oscura y la fuerza de repulsión cósmica.

\section{Entropía}

La segunda ley de la termodinámica establece que el universo tiende al desorden; es decir, el estado de maxima probabilidad, lo que se conoce como Entropia del universo, que a su vez determina la dirrección del tiempo cósmico.
Las particulas virtuales de antimateria neutralizan el tiempo y en consecuencia la Entropía.

en la singular cósmica la entropía se anula totalmente y por consiguiente el universo se encuentra en un estado completamente ordenado.

\section{Ecuaciones de campo modificadas}

Las ecuaciones de campo de la relatividad general están dadas por:

$$
R_{u v}-\frac{1}{2} R g_{u v}=\frac{8 \pi G}{c^{4}} T_{u v}
$$

Donde Ruv son las componentes del tensor de curvatura de Ricci, $\mathrm{R}$ el escalar de curvatura de Ricci, guv las componentes del tensor métrico que permite medir distancias en el espaciotiempo curvo y Tuv las componentes del tensor energía - momento de la materia que crea el campo, que considera la energía y la presión de la materia considerada como un fluido de gas, que reemplaza a la densidad $\rho$ en la ecuación de Poisson.

Estas ecuaciones de campo están basadas en la ecuación de Poisson, y hemos visto que esta ecuación se modifica por efecto de las partículas virtuales de antimateria, de manera que debemos introducir esta modificación, cuyas segundas derivadas no son lineales, a diferencia de las ecuaciones relativistas, en las ecuaciones de campo de Einstein, obteniéndose:

$$
R_{u v}-\frac{1}{2} R g_{u v}=\frac{8 \pi G}{c^{4}}\left(1-\frac{8 \pi G \rho r^{2}}{9 c^{2}}\right) e^{-\frac{4 \pi G \rho r^{2}}{3 c^{2}}} T_{u v}
$$

O bien:

$$
R_{u v}-\frac{1}{2} R g_{u v}=\frac{8 \pi G}{c^{4}} \mathcal{T}_{u v}
$$

Siendo Tuv el tensor energía - momento modificado, el cual se manifiesta en la formación de un agujero negro. Cuando $r=r_{0}=0$, la divergencia es nula. 
Una estrella varias veces más masiva que el sol ejerce una presión interna que contrarresta la gravedad.

A medida que la estrella pierde su combustible la presión disminuye y la gravedad comprime la estrella.

Cuando el combustible se agota la presión desaparece pero surge una nueva presión como consecuencia del principio de exclusión de Pauli, que establece que dos fermiones no pueden ocupar el mismo estado cuántico.

En una estrella masiva la gravedad es más fuerte que la presión ejercida por el principio de exclusión y los electrones se unen a los protones, emitiendo neutrinos, dando origen a las estrellas de neutrones y posteriormente a una estrella de quarks.

Si bien, de acuerdo al modelo propuesto, la gravedad comienza a disminuir a escalas muy pequeñas, la masa es tan grande que la estrella termina colapsando finalmente en un agujero negro.

El resultado final es en consecuencia una masa puntual cuya energía gravitatoria es su energía intrínseca $\mathrm{Mc}_{2}$ con signo negativo.

\section{Experimento propuesto}

Una de las conclusiones que se desprende de las ecuaciones gravitatorias del modelo propuesto es que tratándose de masas diferentes la atracción gravitatoria con que se atraen una a la otra son también diferentes, a diferencia de los modelos newtoniano y relativista, es decir:

$$
\boldsymbol{F}_{M_{m}} \neq \boldsymbol{F}_{m_{M}}
$$

Partiendo de las respectivas ecuaciones de estas fuerzas aparentes podemos encontrar experimentalmente la relación entre ambas fuerzas y el valor exacto de $\mathrm{G}$, así como la demostración experimental del modelo propuesto:

$$
\begin{gathered}
\frac{\boldsymbol{F}_{M_{m}}}{\boldsymbol{F}_{m_{M}}}=e^{-\frac{G(M-m)}{c^{2} r}} \\
G=-\ln \left[\frac{\boldsymbol{F}_{M_{m}}}{\boldsymbol{F}_{m_{M}}}\right] \frac{c^{2} r}{M-m}
\end{gathered}
$$

Los experimentos con diferentes valores de $\mathrm{M}, \mathrm{m}$ y r para determinar una diferencia entre ambas fuerzas deben ser extremadamente precisos y deben dar siempre el mismo resultado para G.

Otra manera de demostrar experimentalmente la validez del modelo propuesto es mediante la observación astronómica de las velocidades angulares de planetas y estrellas.

\section{REFERENCIAS BIBLIOGRÁFICAS}

Alexandrov, A.d. Komologorov, A.n. Laurentiev, M.a. y otros. La matemática: su contenido, métodos y significado. Tomo. I, II y III. Alianza Editorial, S.A. Madrid, 1981.

Álvarez Vita, Enrique. Universos de materia y antimateria. Revista Tradición, Año XIII, N 13, Universidad Ricardo Palma. Lima, 2013.

Álvarez Vita, Enrique. Un esbozo sobre la unificación de las fuerzas fundamentales de la naturaleza y sus implicaciones filosóficas. Revista Evohé, Año III, N 3, Revista Villarrealina de Filosofía. Lima, 2014.

Álvarez Vita, Enrique. Universos fractales de materia $y$ antimateria y el neutrovacío. Revista Ciencia y Desarrollo, volumen 18, número 1, enero - junio 2015. Universidad Alas Peruanas. Lima 2015.

Calcina, Esly Abner. Agujeros negros. Informe de Tópicos de Investigación II, Facultad de Ciencias, Universidad Nacional de Ingeniería. Lima, 2014.

Davis, Paul. Superfuerza. Salvat Editores S.A. Barcelona, 1985.

Davis, Paul. La frontera del infinito. Salvat Editores, S.A. Barcelona, 1985. 
De la Torre, Lorenzo. Elementos de relatividad. Editorial Universidad de Antioquia. Medellín, 2008.

Einstein, Albert. El significado de la relatividad. Espasa - Calpe. Madrid, 2008.

Feynman, Richard. QED: La teoría extraña de la luz y de la materia. Prensa de la Universidad de Princeton. Princeton, 1988.

Hawking, Stephen. Historia del tiempo. Editorial Grijalbo, S.A. Bogotá, 1989.

Enrique Álvarez Vita

Email: ejualvi@hotmail.com 